\title{
Exploring anti-malarial potential of FDA approved drugs: an in silico approach
}

\author{
Gayatri Ramakrishnan ${ }^{1,3,4}$, Nagasuma Chandra ${ }^{2}$ and Narayanaswamy Srinivasan ${ }^{3 *}$ [D
}

\begin{abstract}
Background: The critically important issue on emergence of drug-resistant malarial parasites is compounded by cross resistance, where resistance to one drug confers resistance to other chemically similar drugs or those that share mode of action. This aspect requires discovery of new anti-malarial compounds or formulation of new combination therapy. The current study attempts to contribute towards accelerating anti-malarial drug development efforts, by exploring the potential of existing FDA-approved drugs to target proteins of Plasmodium falciparum.

Methods: Using comparative sequence and structure analyses, FDA-approved drugs, originally developed against other pathogens, were identified as potential repurpose-able candidates against $P$. falciparum. The rationale behind the undertaken approach is the likeliness of small molecules to bind to homologous targets. Such a study of evolutionary relationships between established targets and P. falciparum proteins aided in identification of approved drug candidates that can be explored for their anti-malarial potential.

Results: Seventy-one FDA-approved drugs were identified that could be repurposed against P. falciparum. A total of 89 potential targets were recognized, of which about 70 are known to participate in parasite housekeeping machinery, protein biosynthesis, metabolic pathways and cell growth and differentiation, which can be prioritized for chemotherapeutic interventions. An additional aspect of prioritization of predicted repurpose-able drugs has been explored on the basis of ability of the drugs to permeate cell membranes, i.e., lipophilicity, since the parasite resides within a parasitophorous vacuole, within the erythrocyte, during the blood stages of infection. Based on this consideration, 46 of 71 FDA-approved drugs have been identified as feasible repurpose-able candidates against $P$. falciparum, and form a first-line for laboratory investigations. At least five of the drugs identified in the current analysis correspond to existing antibacterial agents already under use as repurposed anti-malarial agents.
\end{abstract}

Conclusions: The drug-target associations predicted, primarily by taking advantage of evolutionary information, provide a valuable resource of attractive and feasible candidate drugs that can be readily taken through further stages of anti-malarial drug development pipeline.

Keywords: Antimalarial agents, Drug repurposing, Drug targets, Plasmodium falciparum, Sequence analysis

\section{Background}

Over the past decade, extensive measures have been undertaken to prevent or cure malaria, resulting in reduction of mortality rates by $60 \%$ [1]. Despite the tremendous progress, malaria continues to be a global burden with 214 million new incidences and about

\footnotetext{
*Correspondence: ns@mbu.iisc.ernet.in

${ }^{3}$ Molecular Biophysics Unit, Indian Institute of Science, Bangalore 560012, India

Full list of author information is available at the end of the article
}

0.4 million deaths in the year 2015 alone, the primary cause of which is the emergence of drug-resistant strains of Plasmodium falciparum. The current anti-malarial regimens include several drugs administered either alone or in combination, which can be grouped based on their drug class, as summarized in Table 1. Albeit moderately effective, the current treatment regimens remain inadequate due to sub-optimal safety profile in some cases, as described in Table 1, and mainly due to emergence of drug-resistant parasites. Anti-malarial drug resistance, mainly contributed by transporters in 
Table 1 Details on current anti-malarial agents

\begin{tabular}{|c|c|c|c|}
\hline Drug name & Drug class & Anti-malarial activity & Side effects \\
\hline Quinine & \multirow[t]{2}{*}{ Cinchona alkaloids } & \multirow{5}{*}{$\begin{array}{l}\text { Accumulates in food vacuoles and } \\
\text { forms toxic haem complexes }\end{array}$} & Side effects include hearing impairment, \\
\hline Quinidine & & & $\begin{array}{l}\text { rashes, vertigo, vomiting and in some cases } \\
\text { neurotoxicity }\end{array}$ \\
\hline Mefloquine & \multirow[t]{4}{*}{ Quinolines and derivatives } & & $\begin{array}{l}\text { Nausea, dizziness, diarrhoea, bradycardia and } \\
\text { neurotoxicity }\end{array}$ \\
\hline Chloroquine & & & May cause psoriasis \\
\hline Amodiaquine & & & $\begin{array}{l}\text { Vomiting, dizziness and in some cases hepatic } \\
\text { disorders }\end{array}$ \\
\hline Primaquine & & $\begin{array}{l}\text { Believed to block oxidative } \\
\text { metabolism in the parasite }\end{array}$ & Anorexia, vomiting, cramps and anaemia \\
\hline Halofantrine & Phenanthrenes and derivatives & $\begin{array}{l}\text { Causes parasite membrane } \\
\text { damage by forming cytotoxic } \\
\text { complexes }\end{array}$ & $\begin{array}{l}\text { Nausea, diarrhoea, itching and high cardiotoxic- } \\
\text { ity }\end{array}$ \\
\hline Sulfadoxine & \multirow{3}{*}{$\begin{array}{l}\text { Benzene and substituted deriva- } \\
\text { tives }\end{array}$} & \multirow[t]{4}{*}{ Inhibit synthesis of folates } & \multirow[t]{2}{*}{ Skin reactions (rare) } \\
\hline Sulfamethoxypyridazine & & & \\
\hline Proguanil & & & Very few: hair loss and mouth ulcers \\
\hline Pyrimethamine & Diazines & & Occasional rashes \\
\hline Tetracycline & \multirow[t]{2}{*}{ Tetracyclines } & \multirow[t]{2}{*}{ Inhibits translation } & - \\
\hline Doxycycline & & & $\begin{array}{l}\text { Depression of bone growth and gastrointestinal } \\
\text { disturbances }\end{array}$ \\
\hline Clindamycin & Carboxylic acids and derivatives & \multirow[t]{2}{*}{ Inhibits protein synthesis } & Nausea, vomiting and cramps \\
\hline Azithromycin & Macrolides and analogues & & May cause angioedema and jaundice \\
\hline Artemisinin & Lipids and lipid-like molecules & $\begin{array}{l}\text { Believed to affect mitochondrial } \\
\text { electron transport chain [46] or } \\
\text { disrupt cellular redox cycling or } \\
\text { inhibition of haem metabolism } \\
\text { [47] }\end{array}$ & Nausea, anorexia, dizziness and neurotoxicity \\
\hline Atovaquone & Naphthalenes & $\begin{array}{l}\text { Affects mitochondrial electron } \\
\text { transport chain }\end{array}$ & May cause rashes, diarrhoea and headache \\
\hline
\end{tabular}

DrugBank (v.4.3) [26]. The drugs highlighted in italics denote anti-bacterials repurposed for use against malaria

the parasite [2], is also compounded by 'cross resistance', where resistance to one drug confers resistance to other chemically similar drugs or those that share mode of action. The current attempt to prevent development of resistance to artemisinin, a potent first-line anti-malarial agent, includes administration of artemisinin-based combination therapy (ACT) [1], i.e., artemisinin with non-artemisinin partners, such as amodiaquine and mefloquine (Table 1). However, resistance to almost all known anti-malarial agents has reportedly emerged [3, 4] limiting combinatorial therapy as well. This necessitates the discovery and development of either new antimalarial agents or unexplored combination of drugs that may not only reduce mortality and morbidity of the disease, but also reduce the risk of resistance to antimalarial drugs.

Identifying new therapeutic uses of existing drugs has become an attractive practicable strategy due to significant advantages in cost and time involved in the drug development pipeline. Indeed, this strategy has been exploited previously, which led to the formulation of anti-malarial treatment regimens that include anti-bacterials such as sulfonamides, tetracyclines, clindamycin and azithromycin [5]. Albeit successful earlier as a part of combination therapy, these anti-bacterials have become inefficient due to emergence of drug-resistant parasites. Recently, high-throughput chemical library screening initiatives have been useful in identification of repurpose-able drugs that await evaluation on their anti-malarial efficacy $[6,7]$. With the lack of adequate anti-malarial treatment regimen and increasing incidences of drug-resistant parasites, exploring existing drugs for use against malaria is gaining rapid importance [8]. The current study makes an effort to contribute towards the same with the use of FDA-approved drugs.

Using the concept of within-target-family selectivity of small molecules [9], i.e., the likeliness of a small-molecule to bind to related targets, potential repurpose-able drug candidates against malaria could be recognized by means of exploration of evolutionary relationships between targets of FDA-approved 
drugs and $P$. falciparum proteins. An initial filter to exclude drugs known to target human proteins aided in elimination of drugs with undesirable pharmacological potential. This drug-target identification methodology facilitated recognition of 89 P. falciparum proteins which could serve as targets for 71 FDA-approved drugs. The drug-target associations thus predicted can serve as a useful resource for experimental endeavours in the light of anti-malarial drug development and drug discovery.

\section{Methods}

\section{Dataset}

Information on gene products encoded in the genome of P. falciparum 3D7 isolate was obtained from PlasmoDB (release 24) [10]. Proteins annotated as pseudogenes were excluded as such entries correspond to genes that apparently lack protein-coding ability and are unlikely to be expressed in the cell. The protein sequences corresponding to 5400 protein-coding genes were thus retrieved from the database. Furthermore, protein expression profiles of the parasite proteins during their developmental stages in human were extracted from the published works of Pease et al. [11], Florens et al. [12] and Le Roch et al. [13] for intraerythrocytic stages (ring, trophozoite, schizont, merozoite), from Lindner et al. [14] for liver stage (sporozoite), and from Silvestrini et al. [15] for the final gametocyte stage. Details on FDA-approved drugs (1634) and the associated protein targets (1573) were obtained from DrugBank (version 4.3), an extensive resource on drug and target information.

\section{Approach to recognize potential drug-target interactions}

The protocol adopted to identify FDA-approved drugs that can be repurposed against proteins in P. falciparum is similar to the protocol employed in an earlier published report on repurposing drugs against Mycobacterium tuberculosis $\mathrm{H} 37 \mathrm{Rv}$ [16]. The exclusion of drugs known to be effective against human proteins and the exclusion of anti-malarial agents currently in use (Table 1) formed the initial step of the approach. Also, only those drugs that hold evidence on pharmacological validation against established targets were considered. Thus, 196 FDA-approved drugs associated with 138 protein targets were considered for subsequent analysis. The likelihood of drugs to be active as potential anti-malarial agents is dependent on the similarity of known targets to $P$. falciparum proteins. To recognize target-related proteins in P. falciparum, an iterative sequence search program, jackhammer availed through HMMER3.0 suite [17], was employed at an E-value cut-off of 0.0001 and five rounds of iteration. An alignment coverage cut-off of $70 \%$ or an alignment encompassing at least one functional and/or structural domain was used as an additional criterion to eliminate unreliable hits characterized by short stretches of alignment.

Once reliable target-related proteins are recognized in P. falciparum, a comparative structural analysis was undertaken to pinpoint probable drug-binding sites in the parasite proteins, based on information on ligandbinding sites in the known targets. Structural information for target proteins and parasite proteins was obtained from PDB (Protein Data Bank). For parasite proteins of no known structure, structural models were retrieved from ModBase [18] or built using MODELLER v.9.14 [19]. The structural models obtained were checked for reliability based on z-DOPE (Discrete Optimized Protein Energy) score [20] $(<0)$, ModPipe Quality Score (MPQS) cut-off of 1.1 and query coverage threshold of $80 \%$ or a query coverage of at least one functional and/ or structural domain. Comparative evaluation of binding sites across established targets and their homologues in $P$. falciparum was pursued depending on the availability of crystal structures of ligand-bound targets. A structural alignment algorithm, TM-align [21], was employed to assess the extent of conservation of residues in local aligned regions between the parasite proteins and corresponding related targets. A TM-score cut-off of 0.5 was used, which typically implies convincing structural similarity of the proteins aligned [22]. In certain cases, a reliable structural model for the $P$. falciparum protein or its domain could not be built, primarily due to abundance of low-complexity regions in the protein and/or presence of non-conserved inserts within the functional domains. Indeed, these features contribute to the pronounced sequence divergence observed in genome of $P$. falciparum [23-25]. Thus, in such cases, the aligned regions of interest between the protein sequences of $P$. falciparum and related targets were manually examined to infer feasibility of drug-target interactions in P. falciparum. Similarly, in the absence of crystal structures of established targets, potential drug-target interactions in the parasite proteins were conjectured on the basis of alignment of functionally relevant regions.

\section{Results}

Plasmodium falciparum homologues of established targets of FDA-approved drugs were identified using sequence and structural analyses described in "Methods". If $P$. falciparum homologue of a known drug target could be identified, the drug concerned is an attractive possibility for anti-malarial activity.

\section{Potential repurpose-able drug candidates}

A total of 71 FDA-approved drugs, predominantly constituted by antibacterial agents (56), were recognized as repurpose-able candidates against $89 P$. falciparum 
proteins. The complete list of predicted drug-target associations is provided in Additional file 1. At least five of the anti-malarial drugs listed in Table 1 are anti-bacterial and have been repurposed for use against malaria. Based on hierarchical, rule-based, structural classification of drugs, as obtained from DrugBank [26], the 71 drugs could be grouped into 22 drug classes. Figure 1 outlines the distribution of 71 drugs under the 22 classes, along with number of predicted parasite targets associated with each drug class. The predominance of antibacterial agents, represented as green coloured bars, can be readily inferred from the Figure. Notably, a number of instances can be observed in the Figure, which corresponds to drugs with polypharmacological potential, i.e., the ability to act on multiple targets. The most striking example in the Figure is that of two antibacterial drugs (DB00410: mupirocin, DB00548: azelaic acid) under the class 'Fatty acyls' which have been predicted to target 21 parasite proteins; of which nine parasite proteins involved in aminoacylation are potential targets for mupirocin and 12 metabolic proteins of the parasite are potential targets for azelaic acid.

Azelaic acid is a naturally occurring compound produced by Malassezia furfur, and is approved for use as a topical antibacterial agent. This drug is believed to inhibit several metabolic enzymes in bacteria, including tyrosinase, mitochondrial enzymes of the respiratory chain, thioredoxin reductase, 5 - $\alpha$-reductase and DNA polymerase. Indeed, the 12 potential targets recognized in $P$. falciparum belong to these categories of oxido-reductive enzymes, four of which are localized in a vital organelle of the parasite, the apicoplast (highlighted in Table 2). Considering the ability to target multiple metabolic enzymes, azelaic acid forms an attractive repurpose-able candidate against $P$. falciparum. Such polypharmacological drugs have the ability to stand tolerance against development of drug resistance in malarial parasites, and indeed are of immense interest in rationalizing anti-malarial drug discovery and development efforts. Table 2 summarizes the details on the predicted targets of azelaic acid.

Mupirocin, an antibiotic isolated from Pseudomonas fluorescens, is one of the widely used bacteriocidal agent known to inhibit isoleucyl-tRNA synthetase (also known as isoleucyl-tRNA ligase). Recently, a study demonstrated the potent inhibitory activity of mupirocin against bloodstage $P$. falciparum [27]. Despite its effectiveness, mupirocin is unfit to serve as a lead for anti-malarial therapy as it is restricted to topical usage due to rapid hydrolysis in body fluids. Nevertheless, non-hydrolysable mupirocin analogues with evidence on inhibitory activity can be explored for their potential use against $P$. falciparum. Indeed, two analogues of mupirocin, CHEMBL38412 and CHEMBL315230, were identified from an earlier published study [16] where two-step in silico screening (two-dimensional followed by three-dimensional molecular similarity calculations) was employed on a set of FDA-approved drugs against a database of ChEMBL compounds [28, 29], which hosts exhaustive details on drug-like bioactive compounds largely extracted from literature and other databases. Of the two mupirocin analogues CHEMBL38412 and CHEMBL315230, which are experimentally verified inhibitors of isoleucyl-tRNA

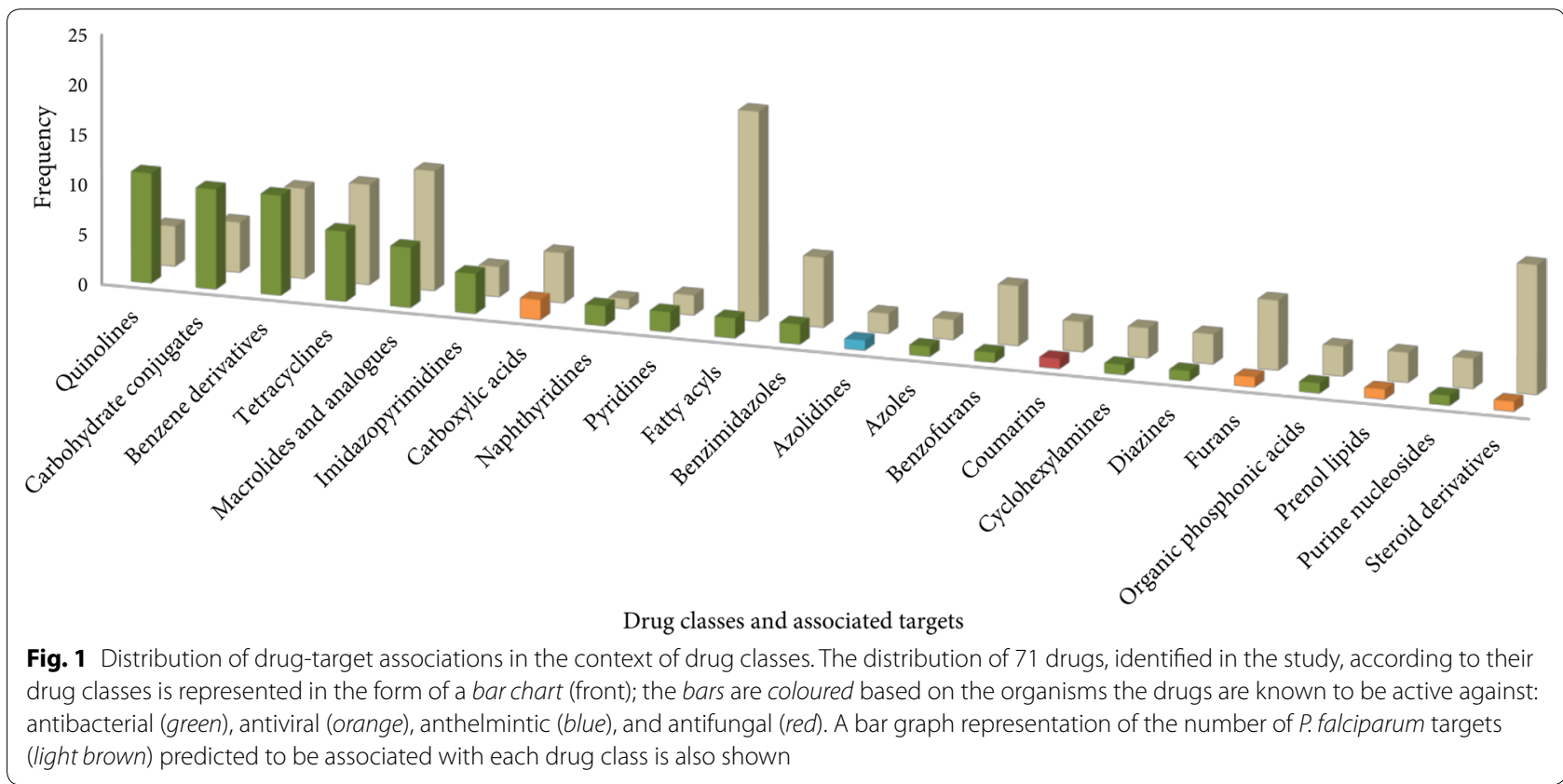




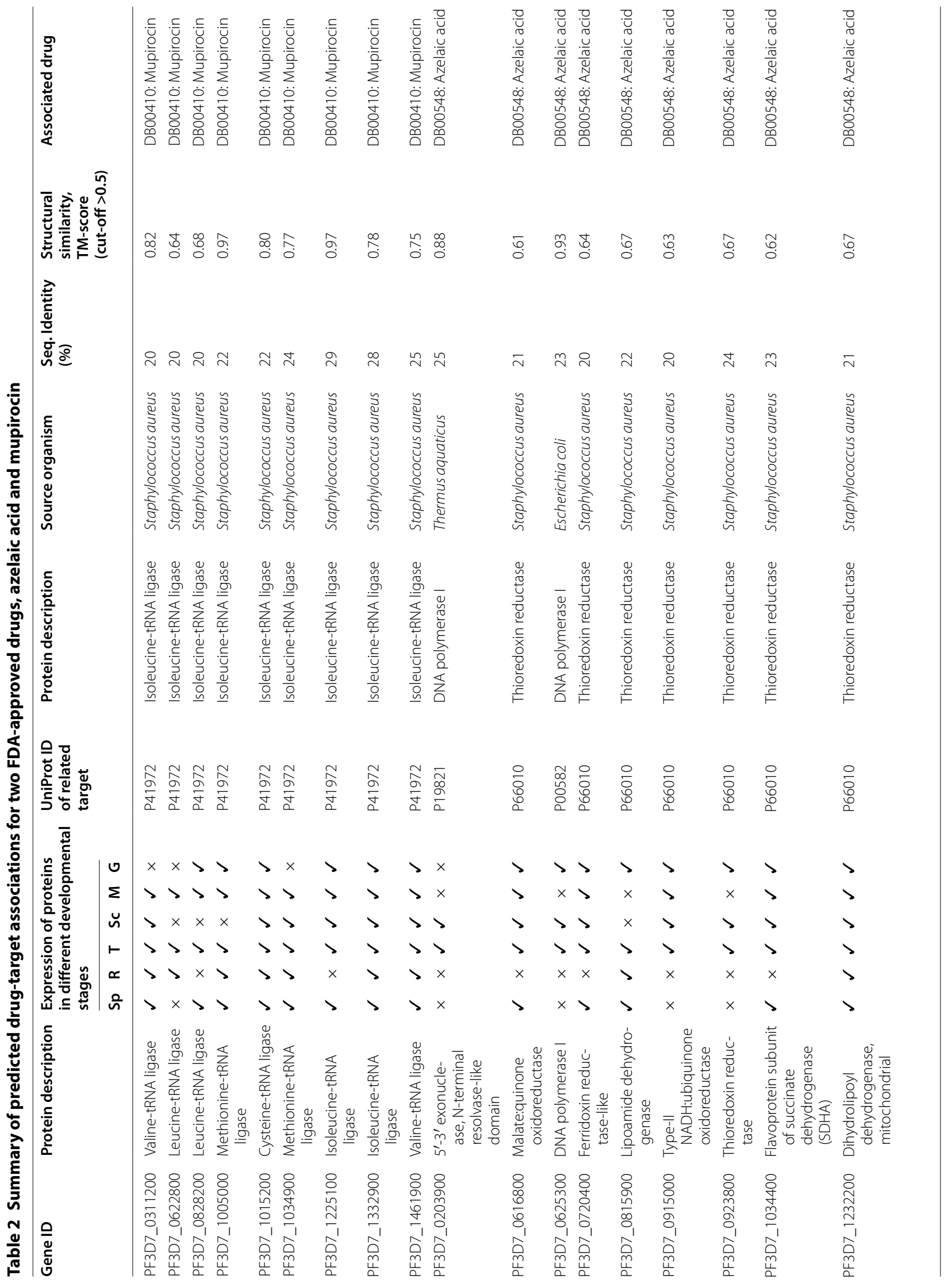




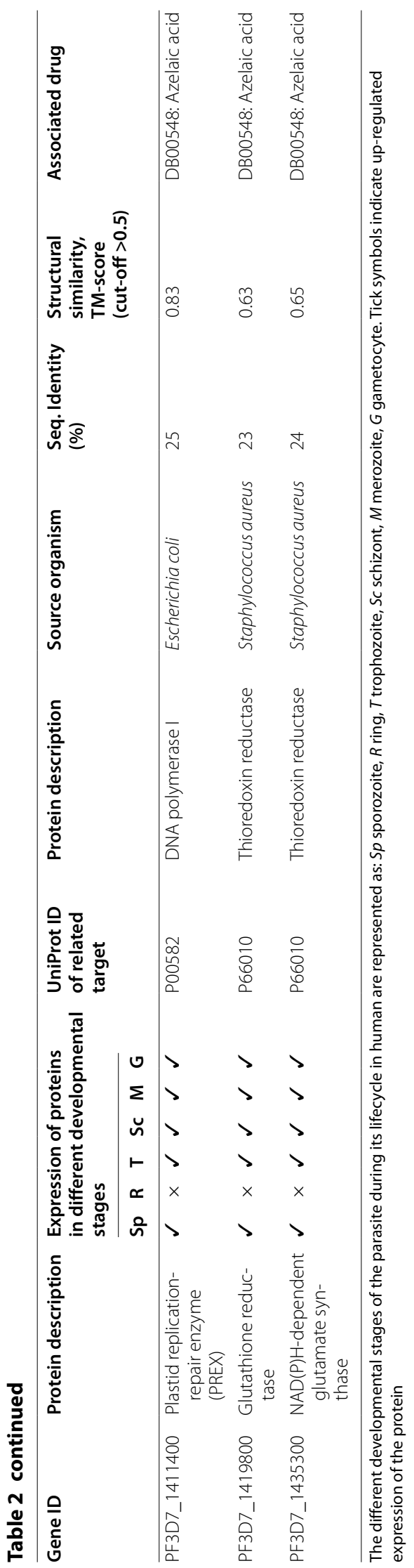


synthetase [30, 31], CHEMBL38412 is reportedly a nonhydrolysable analogue of mupirocin and exhibits similar antibacterial activity in vitro as mupirocin [30]. Elucidations on binding site and binding pose of CHEMBL38412 can be readily inferred based on structural similarity between mupirocin-bound isoleucyl-tRNA synthetase and related parasite proteins (Table 2 and Additional file 2: Figure S1) and the extent of molecular similarity between the drug and its analogue as illustrated in Fig. 2. Based on reasonable structural similarity amongst related proteins as well as the compounds, the mupirocin analogue CHEMBL38412 forms an attractive lead and thus can be investigated further for its anti-malarial activity.

Several other drug classes corresponding to drugs with possible polypharmacological potential, as delineated in Fig. 1, can be investigated further. Figure 1 also illustrates populated drug classes including 'Quinolines', 'Carbohydrate conjugates', 'Benzene derivatives' and 'Imidazopyrimidines' with relatively fewer predicted targets associated with them. Indeed, this observation implies the possibility of combination therapy. But the risk of cross-resistance exists, where resistance to one drug may confer resistance to other chemically similar drugs or those drugs that share similar mechanism of action. Reports on tetracycline and doxycycline resistance, alone or in combination with other drugs, limits the possibility of using approved drugs chemically similar to tetracycline. Seven such drugs (DB00595: oxytetracycline, DB01301: rolitetracycline, DB00618: demeclocycline, DB00256: lymecycline, DB00453: clomocycline, DB01017: minocycline, DB00560: tigecycline) in the drug class 'Tetracyclines' were recognized in the current analysis as potential drug candidates against ten potential parasite targets (Fig. 1). Thorough experimental investigations are necessary to ascertain the likelihood of these drugs as repurpose-able candidates.

\section{Drug targets recognized in Plasmodium falciparum}

The 89 potential targets identified in P. falciparum could be categorized under six functional classes, including<smiles>CCO[C@@H](C)[C@H](C)[C@@H]1O[C@H]1C[C@H]1CO[C@@H](C/C(C)=C/C(=O)OCCCCCCCCC(=O)O)[C@H](O)[C@@H]1O</smiles>

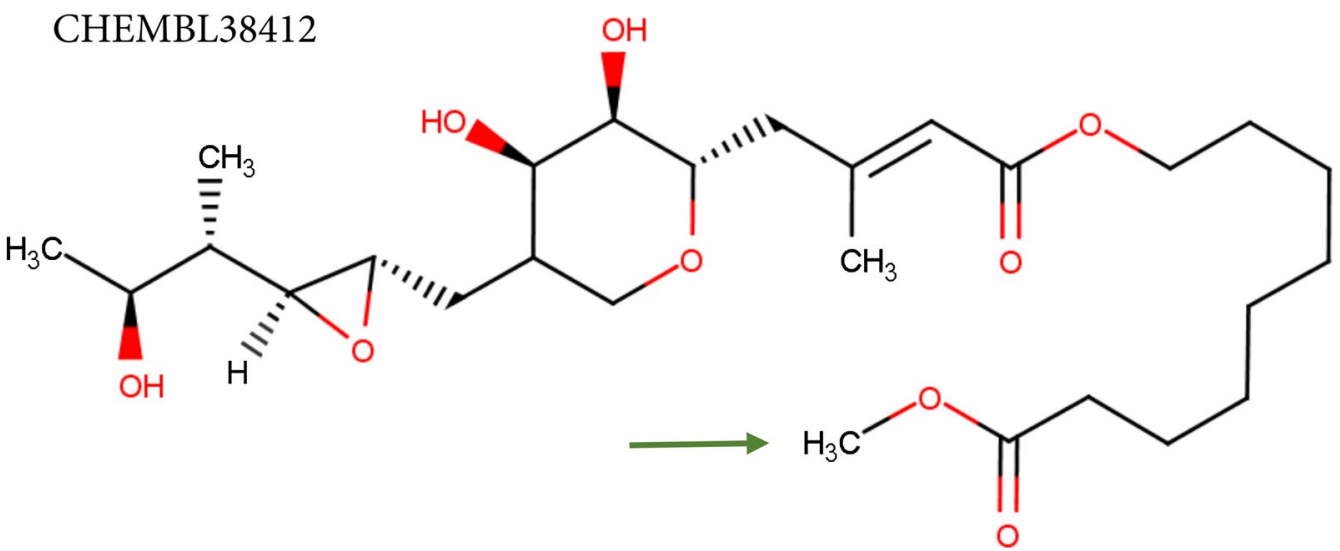

Fig. 2 Mupirocin and its analogue. Two-dimensional chemical representations of the drug mupirocin and its analogue CHEMBL38412 is shown. The presence of non-hydrolysable ester group at C1 position in CHEMBL38412 is depicted with an arrow in green. This figure was generated using Marvin 15.5.18, 2015, ChemAxon [45] 
protein biosynthesis, metabolic processes, DNAdependent activities, microtubule-associated processes, transport and proteolytic activities, most of which are expressed in all six life-cycle stages of infection in human host. Figure 3 illustrates the distribution of predicted targets across the six life-cycle stages of $P$. falciparum binned under six functional categories. As shown in Fig. 3, 38 of 89 predicted targets participate in cellular protein synthesis, which are predominantly expressed in all six life-cycle stages of the parasite. This category includes proteins participating in aminoacylation and protein translation, essential for housekeeping activities. Other proteins of parasite housekeeping machinery including DNA polymerases, DNA topoisomerases, DNA gyrases, microtubules and structural constituents of cytoskeleton, qualify as attractive targets as these $P$. falciparum proteins expressed in all six life-cycle stages during infection in humans play a significant role in regulating rate of translation, cell growth and cell development $[12,23]$. Thus, targeting such parasite proteins (64) belonging to the functional classes 'Protein biosynthesis', 'DNA-dependent processes' and 'Microtubule-associated'
(Fig. 3) can impede growth and transition between different developmental stages of the parasite. Based on the current analysis, a list of 46 drugs were identified, most of which are antibacterial agents, predicted to target 64 proteins involved in parasite housekeeping machinery (Additional file 1).

An important characteristic of $P$. falciparum is its ability to undergo vast re-organization of metabolic make-up during the course of its life cycle in multiple host environments. Inhibition of critically important metabolic enzymes can be detrimental for the parasite's survival, one of which is the glycolytic enzyme lactate dehydrogenase $(p f \mathrm{LDH})$ essential for intra-erythrocytic growth of the parasite [32], recognized as a target for the drug nitrofural (DB00336) in the current analysis. Studies on pfLDH have reported a distinct nature of NADH binding pocket [32] where the anti-malarial chloroquine is believed to bind competitively [33]. Nitrofural, a potent antibacterial agent, is known to inhibit several bacterial enzymes involved in aerobic and anaerobic degradation of glucose and pyruvate. Crystal structure of nitrofural-bound nitroreductase enzyme in Escherichia coli

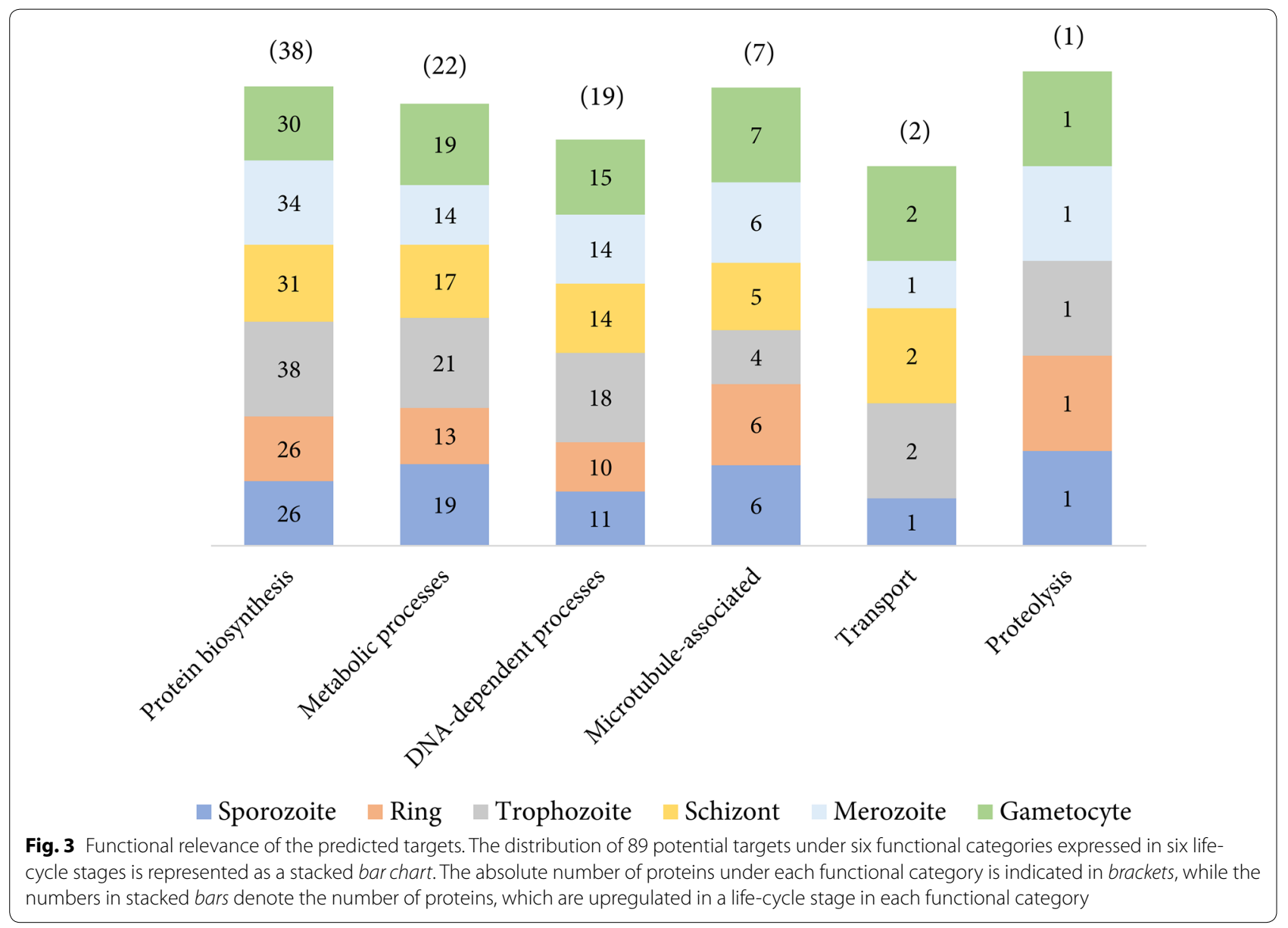


demonstrated the accommodation of the drug in the cofactor binding site [34]. Thus, the cofactor binding site in the crystal structure of $p f \mathrm{LDH}$, i.e., the NADH binding pocket, along with adjacent solvent exposed cavities, were used as receptor grids to dock nitrofural using Glide (Grid-based ligand docking with energetics) [35-37] and assess the predicted binding energy and binding pose of the approved drug. Using Glide XP (extra precision) scoring function [37], the predicted binding site of the drug nitrofural was evaluated. Figure 4 illustrates the docked pose of nitrofural, achieved with a best possible energy score of $-6.97 \mathrm{kcal} / \mathrm{mol}$, at the NADH binding pocket of $p f \mathrm{LDH}$. The docked drug was identified to make contacts with critical residues of $p f \mathrm{LDH}$ that are involved in cofactor binding, including Asp53 and Thr97 (Fig. 4c, d), implying the likeliness of nitrofural in inhibiting the glycolytic enzyme of the parasite. In addition to $p f \mathrm{LDH}$, six $P$. falciparum metabolic proteins, including malate dehydrogenase (PF3D7_0618500), lactate dehydrogenase (PF3D7_132520), lipoamide dehydrogenase (PF3D7_0815900), thioredoxin reductase (PF3D7_0923800), dihydrolipoyl dehydrogenase (PF3D7_1232200), and glutathione reductase (PF3D7_1419800), several of which are essential for the parasite's growth during intra-erythrocytic development stages, were recognized as potential targets

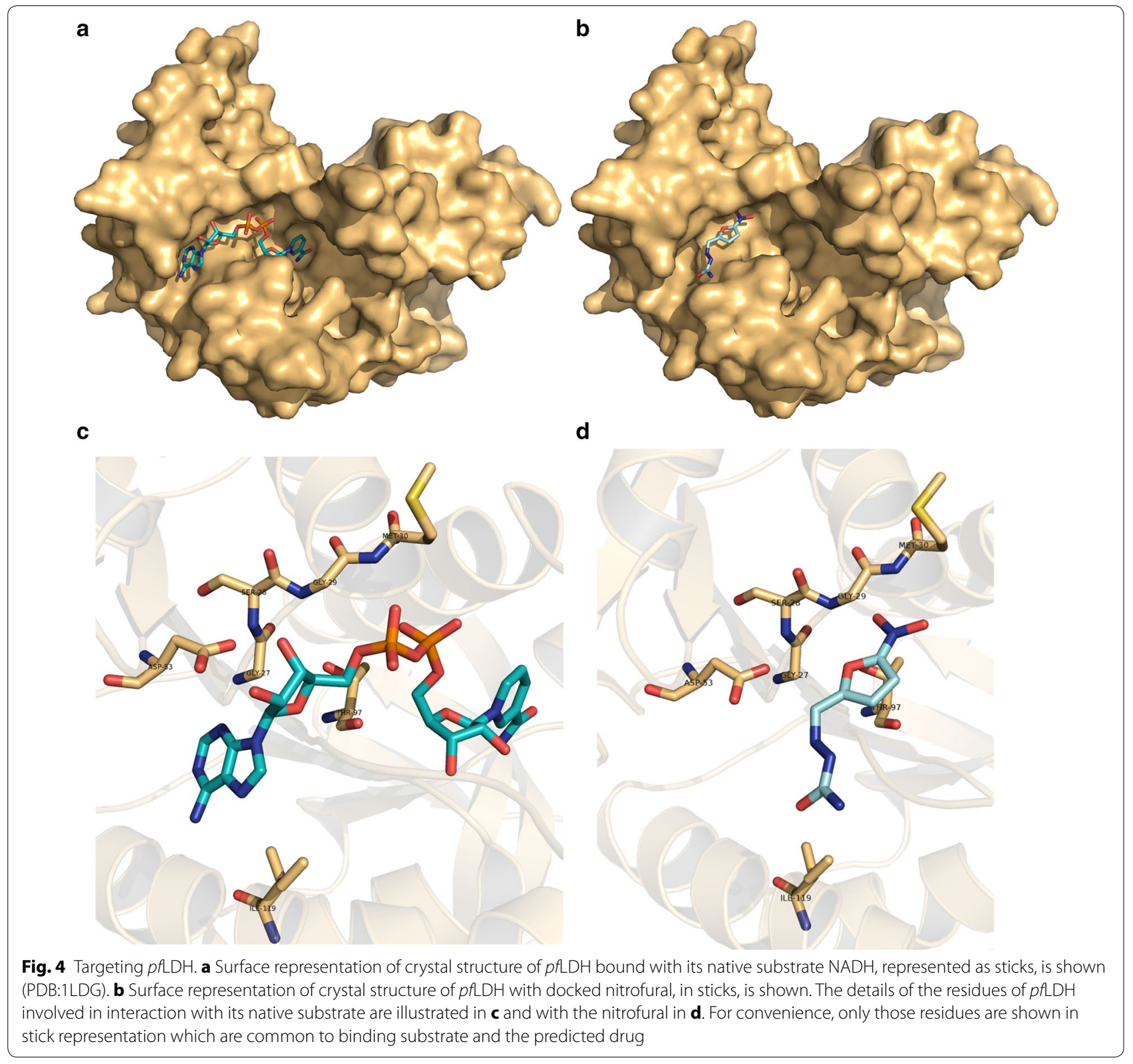


for nitrofural. Considering such a polypharmacological potential of nitrofural, further experimental investigations on this FDA-approved drug can reveal insights into its usefulness as an anti-malarial agent. Other metabolic enzymes identified as targets include enoyl-acyl carrier reductase, dihydropteroate synthetase, thioredoxin reductase, bifunctional dihydrofolate reductase-thymidylate synthase, and other oxido-reductive enzymes which have been regarded as attractive targets for anti-malarial drug development [38], many of which are expressed in all six stages of the parasite's life cycle (Fig. 3). The identification of such pharmaceutically relevant metabolic proteins justifies the strength of the approach used and thus, the feasibility of approved drugs predicted to target such proteins can be further investigated.

Plasmodium falciparum comprises a relict-plastid, termed as the apicoplast, which is indispensable for the parasite's growth and typically contains a range of metabolic pathways and housekeeping processes that differ from those in human hosts [39]. Thus, parasite proteins pertaining to the apicoplast present ideal strategies for drug design. Of 89 predicted targets, 29 were identified as constituents of apicoplast -8 metabolic enzymes and 21 involved in housekeeping machinery. These entries are highlighted in Additional file 1. Information on subcellular localization of other parasite proteins was obtained from Malaria Metabolic Pathways database [40]. Figure 5 illustrates the distribution of number of predicted targets in terms of their subcellular localization in $P$. falciparum (inner ring), along with information on upregulated, intra-erythrocytic stage-specific expression of associated proteins (outer ring). As shown in Fig. 5, more than half of the predicted $P$. falciparum targets are localized in subcellular organelle: nucleus, apicoplast and mitochondrion.

While recognition of proteins essential for the growth and survival of the parasite as targets gains significance, targeting such proteins may be challenging as the parasite resides within the erythrocytes, and additionally, within a protective encasing of parasitophorous vacuole, during blood stages of infection. To reach a target localized in an organelle in the parasite during its intra-erythrocytic development stages, a drug is required to permeate at least four membranes: erythrocyte cell membrane $>$ parasitophorous vacuole membrane $>P$. falciparum cell membrane >organellar membrane. It is thus of importance to consider lipophilicity of the drugs, i.e., the ability of the compounds to dissolve in lipophilic or non-aqueous solutions, which in turn determines the ADMET (absorption, distribution, metabolism, excretion, toxicity) properties of the drug and the likelihood of its therapeutic success [41]. Lipophilicity is typically measured in terms of logarithm of partition coefficient $(\log \mathrm{P})$, which is the ratio of concentrations of a compound between two phases:non-aqueous:octanol and aqueous:water. According to Lipinski's rule of five [42], $\log \mathrm{P}$ value of an active compound should be no more than five, i.e., a concentration difference of 1:100,000 between water and octanol phases. A desirable compound should neither be too lipophilic $(\log P>5)$ nor be too hydrophilic (low or negative $\log \mathrm{P}$ ), for adequate absorption or permeation across cellular membranes. Information on experimentally determined $\log \mathrm{P}$ values of $71 \mathrm{FDA}$-approved drugs was obtained from DrugBank. Forty-six of 71 drugs were recognized which satisfied the $\log P$ value criterion in an optimal range of $(0-5)$. These drugs are speculated to be active against $P$. falciparum during the symptomatic blood stages of infection, and therefore can be prioritized to formulate chemotherapeutic regimen against malaria. Table 3 summarizes the details of 46 FDA-approved drugs that have the potential to influence anti-malarial drug development. Several of the 46 practicable set of drugs, approved for use against other pathogenic infections, have been exploited for their use as anti-malarial agents, examined either in vitro or in vivo (Table 3). Such concurring instances justify the strength of the protocol followed.

The predictions were compared with previously published studies on recognizing anti-malarial potential of approved drugs [8, 43]. Nine drug-target associations were identified pertaining to apicoplast proteins and anti-tubercular drugs (rifamixin, rifampicin) concurring with the predictions made in the current study which, in addition to the set of drugs mentioned in Table 3, can be readily taken through further stages of anti-malarial drug development pipeline.

\section{Conclusion}

An established concept of within-target-family selectivity of small molecules has been pursued which led to the recognition of 71 FDA-approved drugs that can be repurposed against 89 P. falciparum proteins. The framework on drug-target identification methodology established in an earlier published study [16], served as a guiding tool for the workflow adopted. An initial step on exclusion of drugs active against human proteins, minimize the chance of obtaining drugs of undesirable pharmacological potential. Recognition of polypharmacological drugs, capable of targeting multiple proteins, are of great interest for parasites such as P. falciparum, as such drugs can stand tolerance against development of drug resistance. Indeed, the majority of the drugs identified have the potential to target multiple parasite proteins. About 70 of 89 potential targets identified in the current analysis, are known to participate in parasite housekeeping machinery, protein biosynthesis, metabolic pathways, 


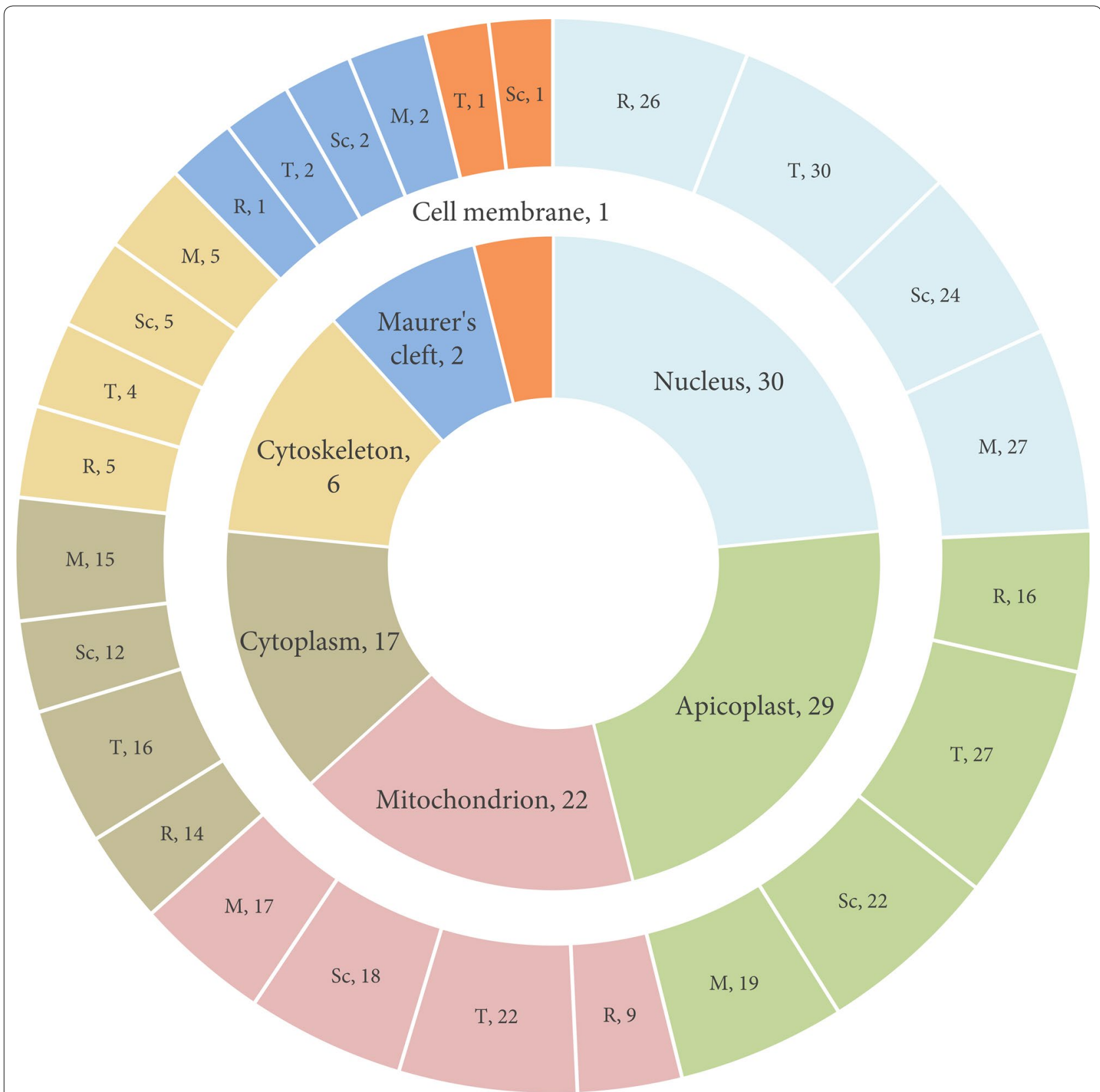

Fig. 5 Subcellular localization of predicted targets. The distribution of predicted targets based on their subcellular localization is delineated in the inner ring. The outer ring denotes number of associated proteins with up-regulated expression in each of the intra-erythrocytic stages, i.e., $R$ ring, $T$ trophozoite, Sc schizont and $M$ merozoite

and cell growth and differentiation. The drug-target associations corresponding to such parasite targets of interest can be investigated further for therapeutic relevance. While the approach undertaken provides a set of promising drug candidates and potential P. falciparum targets, it is limited in terms of evaluation of quantitative drugtarget selectivity. Investigations focused on pharmacokinetics and pharmacodynamics of drugs thus form a pre-requisite to assess the risk of possible drug-target toxicity in humans, in addition to investigations on feasibility of existing drugs as potential anti-malarials.

Considering the intra-erythrocytic growth of $P$. falciparum during the course of infection, an attempt has been made to prioritize the predicted repurpose-able FDAapproved drugs on the basis of their ability to permeate cell membranes, i.e., lipophilicity, since the parasite 
Table 3 Details on 46 FDA-approved drugs that are likely to be active against Plasmodium falciparum during the course of infection

\begin{tabular}{|c|c|c|c|c|}
\hline Drug name & DrugBank ID & Category & Drug class & $\log P$ \\
\hline DB00199 & Erythromycin [48] & Antibacterial & Macrolides and analogues & 3.06 \\
\hline DB00218 & Moxifloxacin & Antibacterial & Quinolines & 2.9 \\
\hline DB00250 & Dapsone $[49]^{a}$ & Antibacterial & Benzene derivatives & 0.97 \\
\hline DB00256 & Lymecycline & Antibacterial & Tetracyclines & 0.3 \\
\hline DB00263 & Sulfisoxazole [50] & Antibacterial & Benzene derivatives & 1.01 \\
\hline DB00336 & Nitrofural & Antibacterial & Furans & 0.23 \\
\hline DB00400 & Griseofulvin & Antifungal & Benzofurans & 2.18 \\
\hline DB00410 & Mupirocin [27] & Antibacterial & Fatty acyls & 2.45 \\
\hline DB00426 & Famciclovir & Antiviral & Imidazopyrimidines & 0.6 \\
\hline DB00446 & Chloramphenicol [51] & Antibacterial & Carboxylic acids & 1.14 \\
\hline DB00453 & Clomocycline & Antibacterial & Tetracyclines & 0.2 \\
\hline DB00487 & Pefloxacin [52] & Antibacterial & Quinolines & 0.27 \\
\hline DB00518 & Albendazole [53] & Anthelmintic & Benzimidazoles & 2.7 \\
\hline DB00537 & Ciprofloxacin [52] & Antibacterial & Quinolines & 0.28 \\
\hline DB00548 & Azelaic acid & Antibacterial & Fatty acyls & 1.57 \\
\hline DB00560 & Tigecycline [54] & Antibacterial & Tetracyclines & 0.8 \\
\hline DB00567 & Cephalexin [55] & Antibacterial & Carboxylic acids & 0.65 \\
\hline DB00576 & Sulfamethizole & Antibacterial & Benzene derivatives & 0.54 \\
\hline DB00609 & Ethionamide & Antitubercular & Pyridines & 0.5 \\
\hline DB00615 & Rifabutin [56] & Antitubercular & Macrolactams & 4.1 \\
\hline DB00618 & Demeclocycline & Antibacterial & Tetracyclines & 0.2 \\
\hline DB00730 & Thiabendazole [53] & Anthelmintic & Benzimidazoles & 2.47 \\
\hline DB00817 & Rosoxacin & Antibacterial & Quinolines & 3.0 \\
\hline DB01015 & Sulfamethoxazole [57] & Antibacterial & Benzene derivatives & 0.89 \\
\hline DB01017 & Minocycline [55] & Antibacterial & Tetracyclines & 0.05 \\
\hline DB01044 & Gatifloxacin [58] & Antibacterial & Quinolines & 2.6 \\
\hline DB01045 & Rifampicin [56] & Antitubercular & - & 2.7 \\
\hline DB01051 & Novobiocin [52] & Antibacterial & Coumarins & 4.1 \\
\hline DB01137 & Levofloxacin [58] & Antibacterial & Quinolines & 2.1 \\
\hline DB01155 & Gemifloxacin & Antibacterial & Naphthyridines & 2.3 \\
\hline DB01165 & Ofloxacin [52] & Antibacterial & Quinolines & 0.65 \\
\hline DB01208 & Sparfloxacin [58] & Antibacterial & Quinolines & 2.5 \\
\hline DB01220 & Rifaximin & Antibacterial & Macrolactams & 2.6 \\
\hline DB01256 & Retapamulin & Antibacterial & Prenol lipids & 4.37 \\
\hline DB01298 & Sulfacytine & Antibacterial & Benzene derivatives & 0.055 \\
\hline DB01321 & Josamycin & Antibacterial & Macrolides and analogues & 3.22 \\
\hline DB01361 & Troleandomycin & Antibacterial & Macrolides and analogues & 4.3 \\
\hline DB01581 & Sulfamerazine & Antibacterial & Benzene and derivatives & 0.14 \\
\hline DB01582 & Sulfamethazine & Antibacterial & Benzene and derivatives & 0.89 \\
\hline DB01603 & Meticillin & Antibacterial & Lactams & 1.22 \\
\hline DB01764 & Dalfopristin [59] & Antibacterial & Macrolide lactams & 1.58 \\
\hline DB02703 & Fusidic acid [60] & Antibacterial & Steroid derivatives & 4.42 \\
\hline
\end{tabular}

resides within a parasitophorous vacuole in the erythrocyte. Using experimentally determined values of octanolwater partition coefficient of drugs, it is estimated that 46 of 71 drugs are feasible repurpose-able candidates against
P. falciparum. These approved drugs can be readily pursued further for an experimental follow-up in vivo.

Much of the drugs identified in the study as prospective anti-malarial agents are antibiotics. The 
Table 3 continued

\begin{tabular}{lllll}
\hline Drug name & DrugBank ID & Category & Drug class & LogP \\
\hline DB04576 & Fleroxacin & Antibacterial & - & 0.24 \\
DB06729 & Sulfaphenazole & Antibacterial & Azoles & 1.52 \\
DB06771 & Besifloxacin & Antibacterial & Quinolines & 0.54 \\
DB08604 & Triclosan [61] & Antibacterial & Quinolines & 4.98 \\
\hline
\end{tabular}

The references included in 23 entries are with respect to the reports on experimental evidence of anti-malarial activity of the drugs

a Chloroproguanil-dapsone combination drug Lapdap has been withdrawn from the market after the observation on significant reductions in haemoglobin levels of patients with glucose-6-phosphate dehydrogenase deficiency [62]

b Chloramphenicol is known to be associated with adverse haematological side-effects [63]

anti-malarial potential of antibiotics has been welldocumented in literature [44], several of which require to be administered in multiple doses in combination with other drugs. Owing to their unintended impact on host microbiome, use of multi-dose antibiotics perhaps should be restricted to patients with severe complications due to malaria and must only be considered after carefully weighing results from preclinical investigations and clinical trials. On similar lines, in the context of safety concerns, clues on FDA-approved drugs obtained from the prioritization exercise attempted in this study can be validated through experimental investigations for efficacy, toxicity, safety, and for the promising candidates, also the therapeutic potential.

The present study provides a set of FDA-approved drugs that are likely to target proteins of the malarial parasite. Albeit, consideration of existing approved drugs can be time and cost-efficient in terms of accelerating anti-malarial drug development efforts, it is extremely important to realize that drug safety cannot be assumed for generic drugs. Indeed, it is imperative that safety of drugs for re-use as anti-malarial agents must be reassessed and concluded with a careful assessment of risk-benefit ratio for a given indication and duration of treatment.

\section{Additional files}

Additional file 1. Details on drug-target associations predicted for Plasmodium falciparum.

Additional file 2. Additional Figure.

\begin{abstract}
Abbreviations
ACT: artemisinin-based combination therapy; ADMET: absorption, distribution, metabolism, excretion and toxicity; DOPE: discrete optimized protein energy; Glide: grid-based ligand docking with energetics; MPQS: ModPipe quality score; PDB: protein data bank; pfLDH: lactate dehydrogenase of $P$. falciparum.
\end{abstract}

Authors' contributions

NS and NC conceived and designed the study. GR performed the data analysis and wrote the manuscript. All authors read and approved the final manuscript.

\section{Author details}

${ }^{1}$ Indian Institute of Science Mathematics Initiative, Indian Institute of Science, Bangalore 560012, India. ${ }^{2}$ Department of Biochemistry, Indian Institute of Science, Bangalore 560012, India. ${ }^{3}$ Molecular Biophysics Unit, Indian Institute of Science, Bangalore 560012, India. ${ }^{4}$ Present Address: Division of Genetics, Department of Medicine, Brigham and Women's Hospital and Harvard Medical School, Boston, MA 02115, USA.

\section{Acknowledgements}

The authors thank Prof R Sowdhamini, NCBS for her support in providing access to Schrödinger suite of programs. We thank the anonymous reviewers for their excellent comments and valuable suggestions.

\section{Competing interests}

The authors declare that they have no competing interests.

\section{Availability of data and materials}

The resultant data corresponding to this work are available in the manuscript in the forms of Tables and Additional files 1,2.

\section{Consent to publication}

All the authors consent to publish.

\section{Funding}

This research was supported by Mathematical Biology programme, Department of Science and Technology, Department of Biotechnology, Ministry of Human Resource Development and by Microsoft Inc. We acknowledge funding for infrastructural support from the following programs of the Government of India: DST-FIST, UGC Center for Advanced Study, and the DBT-IISC Partnership Programme. NS is a JC Bose National Fellow.

\section{Publisher's Note}

Springer Nature remains neutral with regard to jurisdictional claims in published maps and institutional affiliations.

Received: 10 November 2016 Accepted: 13 July 2017

Published online: 18 July 2017

\section{References}

1. WHO. World Malaria Report 2014. Geneva: World Health Organization; 2014.

2. Valderramos SG, Fidock DA. Transporters involved in resistance to antimalarial drugs. Trends Pharmacol Sci. 2006;27:594-601.

3. Dondorp AM, Yeung S, White L, Nguon C, Day NP, Socheat D, et al. Artemisinin resistance: current status and scenarios for containment. Nat Rev Microbiol. 2010;8:272-80

4. Jambou R, Le Bras J, Randrianarivelojosia M. Pitfalls in new artemisinin-containing antimalarial drug development. Trends Parasitol. 2011:27:82-90

5. Nzila A, Ma Z, Chibale K. Drug repositioning in the treatment of malaria and TB. Future Med Chem. 2011:3:1413-26. 
6. Yuan J, Cheng KC, Johnson RL, Huang R, Pattaradilokrat S, Liu A, et al. Chemical genomic profiling for antimalarial therapies, response signatures, and molecular targets. Science. 2011;333:724-9.

7. Matthews H, Usman-Idris M, Khan F, Read M, Nirmalan N. Drug repositioning as a route to anti-malarial drug discovery: preliminary investigation of the in vitro anti-malarial efficacy of emetine dihydrochloride hydrate. Malar J. 2013;12:359.

8. Kaiser M, Maser P, Tadoori LP, loset JR, Brun R. Antiprotozoal activity profiling of approved drugs: a starting point toward drug repositioning. PLoS ONE. 2015;10:e0135556.

9. Kruger FA, Overington JP. Global analysis of small molecule binding to related protein targets. PLoS Comput Biol. 2012;8:e1002333.

10. Aurrecoechea C, Brestelli J, Brunk BP, Dommer J, Fischer S, Gajria B, et al. PlasmoDB: a functional genomic database for malaria parasites. Nucleic Acids Res. 2009;37:D539-43.

11. Pease BN, Huttlin EL, Jedrychowski MP, Talevich E, Harmon J, Dillman T, et al. Global analysis of protein expression and phosphorylation of three stages of Plasmodium falciparum intraerythrocytic development. $J$ Proteome Res. 2013;12:4028-45.

12. Florens L, Washburn MP, Raine JD, Anthony RM, Grainger M, Haynes JD, et al. A proteomic view of the Plasmodium falciparum life cycle. Nature. 2002:419:520-6.

13. Le Roch KG, Zhou Y, Blair PL, Grainger M, Moch JK, Haynes JD, et al. Discovery of gene function by expression profiling of the malaria parasite life cycle. Science. 2003;301:1503-8.

14. Lindner SE, Swearingen KE, Harupa A, Vaughan AM, Sinnis P, Moritz RL, et al. Total and putative surface proteomics of malaria parasite salivary gland sporozoites. Mol Cell Proteomics. 2013;12:1127-43.

15. Silvestrini F, Lasonder E, Olivieri A, Camarda G, van Schaijk B, Sanchez M, et al. Protein export marks the early phase of gametocytogenesis of the human malaria parasite Plasmodium falciparum. Mol Cell Proteomics. 2010;9:1437-48.

16. Ramakrishnan G, Chandra NR, Srinivasan N. Recognizing drug targets using evolutionary information: implications for repurposing FDAapproved drugs against Mycobacterium tuberculosis H37Rv. Mol BioSyst. 2015;11:3316-31.

17. Eddy SR. Accelerated Profile HMM Searches. PLoS Comput Biol. 2011;7:e1002195

18. Pieper U, Webb BM, Barkan DT, Schneidman-Duhovny D, Schlessinger A, Braberg H, et al. ModBase, a database of annotated comparative protein structure models, and associated resources. Nucleic Acids Res. 2011;39:D465-74.

19. Sali A, Blundell TL. Comparative protein modelling by satisfaction of spatial restraints. J Mol Biol. 1993;234:779-815.

20. Shen MY, Sali A. Statistical potential for assessment and prediction of protein structures. Protein Sci. 2006:15:2507-24.

21. Zhang Y, Skolnick J. TM-align: a protein structure alignment algorithm based on the TM-score. Nucleic Acids Res. 2005;33:2302-9.

22. Xu J, Zhang Y. How significant is a protein structure similarity with TMscore $=0.5$ ? Bioinformatics. 2010;26:889-95.

23. Gardner MJ, Hall N, Fung E, White O, Berriman M, Hyman RW, et al. Genome sequence of the human malaria parasite Plasmodium falciparum. Nature. 2002:419:498-511.

24. Tyagi N, Swapna LS, Mohanty S, Agarwal G, Gowri VS, Anamika K, et al. Evolutionary divergence of Plasmodium falciparum: sequences, proteinprotein interactions, pathways and processes. Infect Disord Drug Targets. 2009:9:257-71.

25. Mohanty S, Ramakrishnan G, Dave P, Srinivasan N. Analysis of sequence divergence in metabolic proteins of Plasmodium falciparum: implications for remote homology detection. In: Dunn BM, editor. Frontiers in protein and peptide sciences. Emirate of Sharjah: Bentham Science Publishers; 2014. p. 226-72.

26. Wishart DS, Knox C, Guo AC, Shrivastava S, Hassanali M, Stothard P, et al. DrugBank: a comprehensive resource for in silico drug discovery and exploration. Nucleic Acids Res. 2006;34:D668-72.

27. Istvan ES, Dharia NV, Bopp SE, Gluzman I, Winzeler EA, Goldberg DE. Validation of isoleucine utilization targets in Plasmodium falciparum. Proc Natl Acad Sci USA. 2011;108:1627-32.

28. Bento AP, Gaulton A, Hersey A, Bellis LJ, Chambers J, Davies M, et al. The ChEMBL bioactivity database: an update. Nucleic Acids Res. 2014;42:D1083-90.
29. ChEMBL. 2017. https://www.ebi.ac.uk/chembl/. Accessed 20 Jan 2016

30. Klein LL, Yeung CM, Kurath P, Mao JC, Fernandes PB, Lartey PA, et al. Synthesis and activity of nonhydrolyzable pseudomonic acid analogues. Med Chem. 1989;32:151-60.

31. Brown P, Best DJ, Broom NJ, Cassels R, O'Hanlon PJ, Mitchell TJ, et al. The chemistry of pseudomonic acid. 18. Heterocyclic replacement of the alpha, beta-unsaturated ester: synthesis, molecular modeling, and antibacterial activity. J Med Chem. 1997;40:2563-70.

32. Dunn CR, Banfield MJ, Barker JJ, Higham CW, Moreton KM, Turgut-Balik $D$, et al. The structure of lactate dehydrogenase from Plasmodium falciparum reveals a new target for anti-malarial design. Nat Struct Biol. 1996;3:912-5.

33. Read JA, Wilkinson KW, Tranter R, Sessions RB, Brady RL. Chloroquine binds in the cofactor binding site of Plasmodium falciparum lactate dehydrogenase. J Biol Chem. 1999;274:10213-8.

34. Race PR, Lovering AL, Green RM, Ossor A, White SA, Searle PF, et al. Structural and mechanistic studies of Escherichia coli nitroreductase with the antibiotic nitrofurazone. Reversed binding orientations in different redox states of the enzyme. J Biol Chem. 2005;280:13256-64.

35. Friesner RA, Banks JL, Murphy RB, Halgren TA, Klicic JJ, Mainz DT, et al. Glide: a new approach for rapid, accurate docking and scoring. 1. Method and assessment of docking accuracy. J Med Chem. 2004:47:1739-49.

36. Halgren TA, Murphy RB, Friesner RA, Beard HS, Frye LL, Pollard WT, et al. Glide: a new approach for rapid, accurate docking and scoring. 2. Enrichment factors in database screening. J Med Chem. 2004;47:1750-9.

37. Friesner RA, Murphy RB, Repasky MP, Frye LL, Greenwood JR, Halgren TA, et al. Extra precision glide: docking and scoring incorporating a model of hydrophobic enclosure for protein-ligand complexes. J Med Chem. 2006:49:6177-96.

38. Subbayya I, Ray S, Balaram P, Balaram H. Metabolic enzymes as potential drug targets in Plasmodium falciparum. Indian J Med Res. 1997;106:79.

39. Ralph SA, D'Ombrain MC, McFadden Gl. The apicoplast as an antimalarial drug target. Drug Resist Updates. 2001;4:145-51.

40. Ginsburg H. Progress in in silico functional genomics: the malaria metabolic pathways database. Trends Parasitol. 2006;22:238-40.

41. Arnott JA, Planey SL. The influence of lipophilicity in drug discovery and design. Exp Opin Drug Discov. 2012;7:863-75.

42. Lipinski CA, Lombardo F, Dominy BW, Feeney PJ. Experimental and computational approaches to estimate solubility and permeability in drug discovery and development settings. Adv Drug Deliv Rev. 2001:46:3-26.

43. Bispo NA, Culleton R, Silva LA, Cravo P. A systematic in silico search for target similarity identifies several approved drugs with potential activity against the Plasmodium falciparum apicoplast. PLoS ONE. 2013;8:e59288.

44. Schlitzer M. Malaria chemotherapeutics part I: history of antimalarial drug development, currently used therapeutics, and drugs in clinical development. ChemMedChem. 2007;2:944-86.

45. ChemAxon. 2017. https://www.chemaxon.com/. Accessed 20 Jan 2016.

46. Li W, Mo W, Shen D, Sun L, Wang J, Lu S, et al. Yeast model uncovers dual roles of mitochondria in action of artemisinin. PLoS Genet. 2005;1:e36.

47. Cumming JN, Ploypradith P, Posner GH. Antimalarial activity of artemisinin (qinghaosu) and related trioxanes: mechanism(s) of action. Adv Pharmacol. 1997;37:253-97

48. Nakornchai S, Konthiang P. Activity of azithromycin or erythromycin in combination with antimalarial drugs against multidrug-resistant Plasmodium falciparum in vitro. Acta Trop. 2006:100:185-91.

49. Amukoye E, Winstanley PA, Watkins WM, Snow RW, Hatcher J, Mosobo $M$, et al. Chlorproguanil-dapsone: effective treatment for uncomplicated falciparum malaria. Antimicrob Agents Chemother. 1997;41:2261-4.

50. Berman SJ. Chloroquine-pyrimethamine-sulfisoxazole therapy of Plasmodium falciparum malaria. An alternative to quinine. JAMA. 1969;207:128-30.

51. Ruiz-Sanchez F, Quezada M, Paredes M, Casillas J, Riebeling R. Chloramphenicol in malaria. Am J Trop Med Hyg. 1952;1:936-40.

52. Divo AA, Sartorelli AC, Patton CL, Bia FJ. Activity of fluoroquinolone antibiotics against Plasmodium falciparum in vitro. Antimicrob Agents Chemother. 1988;32:1182-6.

53. Skinner-Adams TS, Davis TM, Manning LS, Johnston WA. The efficacy of benzimidazole drugs against Plasmodium falciparum in vitro. Trans R Soc Trop Med Hyg. 1997:91:580-4.

54. Sahu R, Walker LA, Tekwani BL. In vitro and in vivo anti-malarial activity of tigecycline, a glycylcycline antibiotic, in combination with chloroquine. Malar J. 2014;13:414 
55. Pradines B, Rogier C, Fusai T, Mosnier J, Daries W, Barret E, et al. In vitro activities of antibiotics against Plasmodium falciparum are inhibited by iron. Antimicrob Agents Chemother. 2001;45:1746-50.

56. Sousa M, Pozniak A, Boffito M. Pharmacokinetics and pharmacodynamics of drug interactions involving rifampicin, rifabutin and antimalarial drugs. J Antimicrob Chemother. 2008;62:872-8.

57. Petersen E. In vitro susceptibility of Plasmodium falciparum malaria to pyrimethamine, sulfadoxine, trimethoprim and sulfamethoxazole, singly and in combination. Trans R Soc Trop Med Hyg. 1987;81:238-41.

58. Mahmoudi N, Ciceron L, Franetich JF, Farhati K, Silvie O, Eling W, et al. In vitro activities of 25 quinolones and fluoroquinolones against liver and blood stage Plasmodium spp. Antimicrob Agents Chemother. 2003:47:2636-9.
59. Barthel D, Schlitzer M, Pradel G. Telithromycin and quinupristin-dalfopristin induce delayed death in Plasmodium falciparum. Antimicrob Agents Chemother. 2008;52:774-7.

60. Gupta A, Mir SS, Saqib U, Biswas S, Vaishya S, Srivastava K, et al. The effect of fusidic acid on Plasmodium falciparum elongation factor $\mathrm{G}$ (EF-G). Mol Biochem Parasitol. 2013;192:39-48.

61. Surolia N, Surolia A. Triclosan offers protection against blood stages of malaria by inhibiting enoyl-ACP reductase of Plasmodium falciparum. Nat Med. 2001;7:167-73.

62. Luzzatto $L$. The rise and fall of the antimalarial Lapdap: a lesson in pharmacogenetics. Lancet. 2010;376:739-41.

63. Mintzer DM, Billet SN, Chmielewski L. Drug-induced hematologic syndromes. Adv Hematol. 2009:2009:495863.

\section{Submit your next manuscript to BioMed Central and we will help you at every step:}

- We accept pre-submission inquiries

- Our selector tool helps you to find the most relevant journal

- We provide round the clock customer support

- Convenient online submission

- Thorough peer review

- Inclusion in PubMed and all major indexing services

- Maximum visibility for your research

Submit your manuscript at www biomedcentral.com/submit 